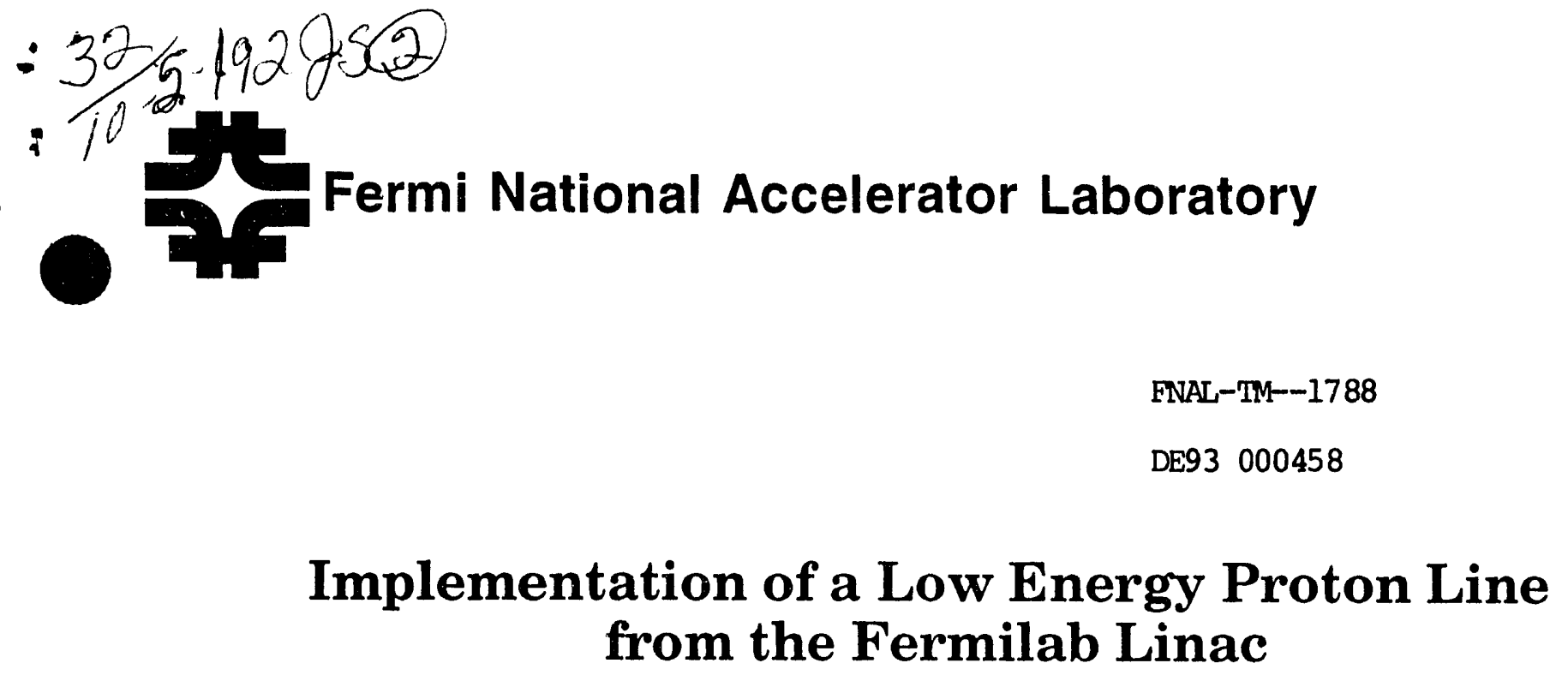

Carol Johnstone

Fermi National Accelerator Laboratory

P.O. Box 500, Batavia, Illinois 60510

July 1992

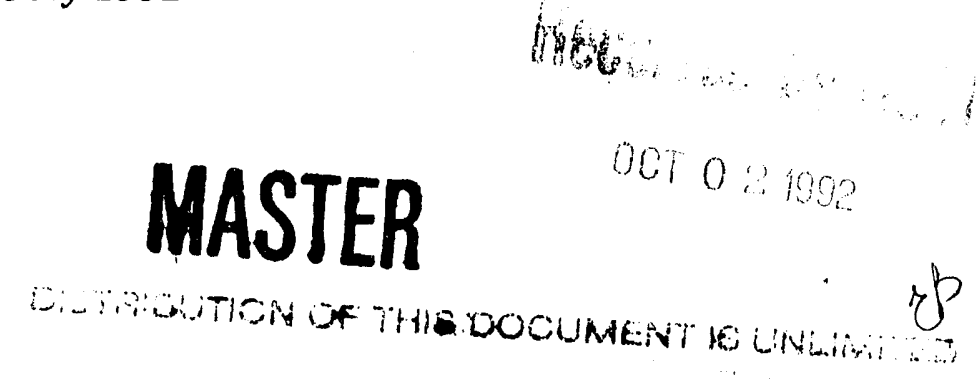




\title{
IMPLEMENTATION OF A LOW ENERGY PROTON LINE FROM THE FERMILAB LINAC
}

\author{
Carol Johnstone \\ Fermi National Accelerator Laboratory, P.O. Box 500, Batavia, IL 60510
}

\begin{abstract}
Two beamlines presently transport the $\mathrm{H}^{-}$beam from the Fermilab Linac. Implementing extraction of some portion of the beam from either line is complicated by the close confines of the enclosure area and the presence of the elements in the existing lines. A new technique has been devised which employs laser stripping of $\mathrm{H}^{-}$to $\mathrm{H}^{0}$ in order to extract a third beam using the existing beamline components in their current configuration. The laser approach has the additional advantages that it provides extreme flexibility in manipulating both the extracted beam pulse intensity and duration through control of the laser's power and pulse length. With the intensity control provided by the laser, a low energy proton line coming out of the Linac, the first of its kind at Fermilab, could be implemented. The line could be multipurpose; it could be used for proton therapy research, detector engineering runs, nuclear physics, accelerator studies, etc. In addition, beam would be available whenever the Linac is runring and not bi subject to the particular program in effect (collider vs. fixed-target, for example).
\end{abstract}

\section{Introduction}

When beam is injected into the Booster Synchrotron for Fermilab's high energy physics (HEP) program, the entire Linac beam pulse is not used. Only the central, goodbeam portion of the Linac beam pulse is transported to the Booster. Typically 8-17 $\mu$ sec, or approximately half or less of the 30- $\mu$ sec-long Linac pulse, is injected into the Booster for the laboratory's physics programs. Unused Linac beam ultimately ends in a high intensity dump. Also, the Linac is capable of producing beam at a $15 \mathrm{~Hz}$ rate, but the HEP program is not able to consume beam at a comparable rate. As a result, a number of Linac cycles remain available.

Given that a significant amount of Linac beam is not used, a third line capable of extracting such beam or exploiting unused Linac cycles, without interfering with $\mathrm{HEP}$, would be a substantive asset to the laboratory, expanding its research and technical capability. A low-energy proton beam derived from the Linac (which is currently a $200-\mathrm{MeV} \mathrm{H}^{-}$ beam, but is soon to be upgraded to $400 \mathrm{MeV}$ ) offers a number of possibilities, particularly since no low-energy proton experimental lines presently exist at Fermilab. A low energy proton line has potential for medical purposes, nuclear experiments, technology transfer, a test beam for both detector calibration and detector engineering runs, or providing a continuous source of beam for accelerator studies and technology. The following sections 
describe the existing beamlines out of the Linac and two schemes for diverting presently unused beam into a new line. One scheme applies the conventional electrostatic separation technique currently in use. The second scheme proposes and explores the power of a new extraction technique based on laser photodetachment of an $\mathrm{H}^{-}$beam.

\section{0-MeV Extraction to the Booster}

Two beamlines have been implemented to handle beam exiting the Fermilab Linac. One line is called the transfer line and transports the central, stable portion of the Linac beam pulse down the chute (see Figure 1) to the Booster Synchrotron. The other, diagnostic line, diverts the remainder of the Linac beam through a spectrometer magnet and into a beam dump. Momentum analysis and some monitoring of the beam is performed in the diagnostic line.

The separation scheme planned for the $400-\mathrm{MeV}$ energy upgrade of the Fermilab Linac is based on the current $200-\mathrm{MeV}$ separation system ${ }^{3}$, which utilizes an electrostatic "chopper" device. The chopper comprises two parallel aluminum capacitor plates $1.43 \mathrm{~m}$ long and separated vertically by 1 inch. It is located just downstream of the last RF tank in the Linac. Before the beam pulse exits the Linac, both plates are charged to $60 \mathrm{kV}$. When Linac extraction begins, the head of the 30- $\mu$ sec-long beam pulse travels unperturbed between the chopper plates, then through a horizontally-focussing quadrupole and into the field-free region of a downstream lambertson. This beam, which represents the first part of the Linac beam pulse, heads down the diagnostic line after exiting the lambertson. Then, at a preset time during the pulse (determined by the amount of beam to be injected into the Booster), the thyratron in the chopper power supply crowbars one capacitor plate to ground. Linac beem is thereby deflected downward by $5 \mathrm{mr}$ and travels off-center through the quadrupole. The off-axis quadrupole field provides another 3 milliradians of downwards dipole kick to the beam. This deflected portion enters the field region of the lambertson. The $10^{\circ}$ horizontal bend of the lambertson causes beam to be deflected into the transfer line and completes the physical separation of the transfer line from the diagnostic line. The two distinct beamlines can be seen emerging from the lambertson (Figure 1). Once the amount of beam required for Booster operation is extracted to the transfer line, the power supply crowbars the other chopper plate, causing the tail of the Linac beam to follow the same path as its head; i.e. along a straightahead trajectory into the diagnostic line. On selected machine cycles (the Linac and Booster are both pulsed at $15 \mathrm{~Hz}$ ), the length of the beam pulse sent to the Booster can be varied by programming the control software to change the crowbar timing. Depending on the application, the length of the beam pulse sent to the Booster can be as short as $1 \mu \mathrm{sec}$ or the entire $30 \mu \mathrm{sec}$ can be injected. Even when all of the Linac beam is injected, space charge and coupled bunch modes cause a significant fraction of the beam to fall out of the Booster, so it is difficult to utilize all of the Linac beam pulse.

To take this unused beam from either the transfer or diagnostic line and direct it into a third beamline is complicated by the fact that much of the enclosure area (Figure 1) is occupied by the elements which already split the Linac beam pulse between the two lines. Adding a beamline and/or altering the transfer or diagnostic line would be difficult in the space available. (Space is restricted because the end of the enclosure is marked by a 
$3 \mathrm{ft}$. by $3 \mathrm{ft}$. concrete-enclosed chute through which the transfer line drops from the Linac elevation to the Booster altitude.) Without a major rework of the two existing lines, or the enclosure, or the high-intensity dump, a third line would have to extract beam from the transfer line. (The only path open to beam extracted from the diagnostic-line would take it across the top of the high-intensity dump ${ }^{1}$, which raises safety concerns.)

To split off beam destined for the transfer line and redirect it into a third beamline requires that the process begin at or near the chopper to avoid encountering either the chute enclosure or the beam dump. If extraction from the tra fer line was attempted downstream of the lambertson, nearly $90^{\circ}$ of horizontal bend would be required (see Figure 1) to avoid these two obstacles. Upstream of the lambertson only two schemes for extraction from the transfer line are feasible: the chopper could be used to kick Linac beam up instead of down on selected Linac cycles ${ }^{4}$, or; the charge of the deflected beam could be changed before the lambertson so that it would not assume the transfer-line trajectory. These two options are discussed in the next two sections.

\section{High Intensity Extraction Using the Chopper}

By reversing the order in which the chopper plates are crowbarred the Linac beam could be deflected up by the same amount it is presently deflected down into the transfer line. Beam would then proceed along the same horizontal trajectory as the diagnosticline beam but angled upwards by $0.46^{\circ}$ (this would require the field-free aperture in the lambertson to be increased from a $1 "$ to a 2 " diameter). Following the lambertson, a vertical septum could be placed just upstream of the horizontal bender, MH1, to complete the separation of the new line. The proposed beamline would then follow the diagnostic line, except that it would be 18" higher, centerline to centerline. The advantage to a vertical displacement would be that quadrupoles and other elements could be added easily to the line, as opposed to competing for the already cramped horizontal space in the current enclosure. The extracted beam would be steered west just above the spectrometer magnet towards a new enclosure. To avoid the dump, a bend of approximately $40^{\circ}-50^{\circ}$ would be required. In order to keep stripping losses below $0.1 \%$, magnetic fields would have to be kept $<7.5 \mathrm{kG}$ for a $400-\mathrm{MeV} \mathrm{H}^{-}$beam. Therefore, either the bend would have to be accomplished using a 3 meter-long dipole, or the $\mathrm{H}^{-}$beam would have to be stripped to protons beforehand, allowing a much shorter, stronger magnet to be used.

In such an extraction scheme beam could not be delivered to the Booster on the same machine cycle. The present thyratron-switched power supply requires $30 \mathrm{~ms}$, or basically the entire intercycle period, to charge the chopper plates. Therefore it could not perform a more complex pulsing scheme than the one described in the previous section. To charge on a microsecond, rather than a millisecond, timescale would require a new power supply capable of delivering two orders of magnitude more current, or $1500 \mathrm{~A}$ at $60 \mathrm{kV}$. The high current of such a supply would be a complication, but a more serious drawback would be that in the few microseconds required to recharge the plates the entire Linac current would strike the steel of the lambertson, thereby creating significant radiation problems. By comparison, crowbarring of one plate occurs in 25 ns so the amount of beam hitting the lambertson during normal operation is a factor of 100 less. Keeping the present chopper power supply, however, would mean that only the beam crcles not used by the Booster 
could be steered into a third beamline. Another limitation to extracting with the chopper would be that the full $35 \mathrm{~mA}$ current of the Linac would have to be delivered to the new line. No intensity moderation would be possible.

Several drawbacks to this scheme become apparent. Beam cannot be injected into the Booster on the selected extraction cycles. The beam extracted would be the full current produced by the Linac. Finally, the much larger hole bored in the lambertson would strengthen higher order harmonics. In particular, skew quadrupole and sextupole components would be enhanced, potentially causing complex matching problems at the Booster.

\section{Variable Intensity Extraction using Laser Neutralization}

Alternatively, extraction could be achieved using the chopper in its standard switching configuration, provided that the deflected beam was neutralized just after traversing quadrupole QT2 (Figure 1). Beam that was neutralized before entering the field region of the lambertson would continue downward by $0.46^{\circ}$, but would not bend west into the transfer line. Instead it would follow the same horizontal trajectory as the diagnostic line, only displaced down with respect to that line. If the neutral beam was stripped to protons by a foil at the exit of the lambertson, then a vertical septum could be inserted upstream of $\mathrm{MH} 1$ to finish the physical separation of the new line from the diagnostic line. The new line would then run below the diagnostic line until it neared the position of the diagnosticline spectrometer magnet, at which point it would have to undergo a $40^{\circ}-50^{\circ}$ bend west to avoid the high-intensity beam dump. This is the same optical and geometrical solution described in the previous section except that the line would lie below, instead of above, the diagnostic line. The separation of the new line from the diagnostic line would again be about 18 ". When the new line eventually crossed over the transfer line, they would be separated by at least $16 "$, center to center.

Neutralizing chopper-deflected beam with a thin foil or wire would not be a feasible approach. Large numbers of protons would unavoidably be produced and these would strike the east side of the lambertson, again creating radiation problems. The wire would have the added disadvantage of only stripping a narrow slice out of the entire Linac phase space. Both the optics and intensity of a beam extracted using a wire would be particularly sensitive to beam jitter.

One elegant solution for stripping a $\mathrm{H}^{-}$beam to $\mathrm{H}^{0}$ without producing protons would be to photoeject the outer electron of $\mathrm{H}^{-}$. The outer electron of $\mathrm{H}^{-}$is bound by only $0.75 \mathrm{eV}$ and its photoejection cross section peaks at about $1.5 \mathrm{eV}$, with a peak width that extends $+/-.5 \mathrm{eV}$. This energy corresponds to an infrared wavelength of around a micron. A common industrial laser such as the high-power, pulsed Nd:YAG laser, which has a photon energy of $1.17 \mathrm{eV}$ and a wavelength of $1.064 \mu \mathrm{m}$, is ideal for photoneutralizing $\mathrm{H}^{-}$to $\mathrm{H}^{0}$.

Unique to the laser stripping approach is the ability to select the intensity of the extracted beam. The entire beam or only a fraction of a percent of the $\mathrm{H}^{-} \mathrm{s}$ can be neutralized. As an example, the power required to strip the entire output of the Fermilab Linac can be calculated as follows. Assuming the laser used is a Nd:YAG laser and the collision is headon in the interaction region, the required photon current is: 


$$
I=A / \sigma\left(v / L_{\text {int }}\right)(1 /(1+\beta))
$$

where $\mathrm{A}$ is the area of the $\mathrm{H}^{-}$beam, $\mathrm{L}_{\text {int }}$ the length of the interaction region, $\sigma$ the photoionization cross secti.n, $v$ the velocity of the beam, and $\beta=v / c$, with $c$ the velocity of light. A realistic value for the area of the $400-\mathrm{MeV} \mathrm{H}^{-}$beam upstream of the lambertson is $.8 \mathrm{~cm}^{2}$. At $400 \mathrm{MeV} \beta$ is .713 and the length of the interaction region available between the chopper and lambertson is 3 meters. At $400 \mathrm{MeV}$, a $1.17 \mathrm{eV}$ photon in the laboratory frame is Doppler shifted to $2.8 \mathrm{eV}$ in the rest frame of the $\mathrm{H}^{-}$ion for a headon collision. At this energy the photoionization cross section ${ }^{5}$ is reduced by a factor of 2 from its peak value-from $4 \times 10^{-17} \mathrm{~cm}^{2}$ to $2.2 \times 10^{-17} \mathrm{~cm}^{2}$. Inserting these values in the above equation gives a current requirement of $1.5 \times 10^{24}$ photons/sec in order to strip all of the $\mathrm{H}^{-}$s. Including the photon energy of $1.17 \mathrm{eV}$ results in an instantaneous laser power of $281 \mathrm{~kW}$. This is within the reach of current technology, where a typical non-amplified, off-the-shelf Nd:YAG achieves $50-100 \mathrm{~kW}$ of instantaneous power, with a 10-30 $\mathrm{Hz}$ firing rate capability (no Q-switching). Amplification or use of a ring-resonator Nd:YAG can increase the power produced to that required for complete stripping.

The photon energy and therefore the laser power required for photoionization is minimized by a headon interaction. At this collision angle the relativistic Doppler shift has the effect of maximizing the observed photon energy in the rest frame of the $\mathrm{H}^{-}$ion. Another substantial reduction in power, the factor of $(1+\beta)^{-1}$ in the equation above, comes from the kinematics of colliding beams. In contrast, this factor becomes $(1-\beta)^{-1}$ when laser and particle beams traverse the interaction region in the the same direction. For applications which do not involve secondary production processes such as proton therapy, nuclear studies, and detector calibration, typically only $10 \%$ or less of the Linac current and microsecond pulse lengths is required, further reducing the laser power by at least an order of magnitude.

The main consideration with using lasers such as the Nd:YAG regards the pulse length. A Q-switched Nd:YAG typically delivers a 30 ns pulse and without the Q-switch the pulse length is milliseconds, neither of which comes close to the appropriate microsecond time window. The millisecond pulse can be chopped to microsecond length using a Pockel cell, but then the majority of the pulse is thrown out and the laser power is unnecessarily large. Changing the pulse length of the laser by redesigning the pulse forming network should not be difficult technically and may represent the most practical solution.

\section{Extraction of Lower-Energy $\mathbf{H}^{-}$Beams}

In both schemes beams with kinetic energies lower than $400-\mathrm{MeV}$ can also be extracted. This would require that one or more of the Linac tanks ${ }^{6}$ be mistimed so that beam is accelerated in the initial tanks and allowed to coast in the remaining ones. (The effect of beam loading and self excitation will have to be calculated.) The fields in the Linac quadrupoles located after the affected tanks would have to be set to values appropriate to the new energy in order to maintain the Linac lattice. Since the Linac quadrupoles are pulsed at $15 \mathrm{~Hz}$, this mode of operation should be relatively straightforward. Likewise the chopper high voltage and QT2's field must match the new energy in order to retain the same downward deflection angle as in $400-\mathrm{MeV}$ operation. Since the Booster cannot 
accept or dispose of beam at energies lower than $400-\mathrm{MeV}$ (after the upgrade takes place), lower-energy beam must be directed into the high-intensity dump. This is also relatively easy by installing a pulsed dogleg to bend beam up and west into the dump. The first pulsed dipole would have to be installed upstream of MV1. Its field would not have to be particularly strong since it only has to bend the $\mathrm{H}^{-}$beam less than a foot up vertically to intersect the diagnostic line (see Figure 1). The second dipole in the dogleg, located at the intersection of the diagnostic and transfer lines, would have to bend beam through a $25^{0}$ angle to intercept the correct trajectory to the dump. This dipole would be smaller than the spectrometer which has to bend through $40^{\circ}$. Its field could be approximately $7.5 \mathrm{kG}$ with a length less than 2 meters. If the seven new rf modules and the transition section (the accelerating, cavities for the $400-\mathrm{MeV}$ Linac Upgrade Project) are mistimed in decreasing sequential order, then the beam energies available for extraction would be 357 , $313,271,229,190,152,116$, and 66 , respectively.

\section{Conclusion}

Using a laser extraction system it is possible to install a variable energy, variable intensity proton beam just downstream of the Linac. A proton beam with an energy that can be varied from 66 to $400 \mathrm{MeV}$ and an intensity that can range from $1 \times 10^{13} \mathrm{ppp}$ to a few protons per pulse would be available on a parasitic basis with HEP. Such a low energy proton line would provide Fermilab with expanded research capabilities, in particular, for nuclear and medical physics experiments.

This paper further shows that there are several drawbacks to chopper extraction, all of which can be effectively addressed by a laser extraction technique. The prominent chopper shortcomings are as follows. First, no beam could be delivered to the Booster during the same cycle that beam was extracted to the new line. A second consideration is that the entire upgrade Linac current of $35 \mathrm{~mA}$ would be delivered to the new line, which would require construction of a new high-intensity dump. The most serious issue regards safety. The radiation and interlock safety system which includes the chopper does not adequately address the problem of limiting dose rates, particularly for medical applications. Without upgrading and increasing the complexity of the current system, the entire $30-\mu$ sec Linac berm pulse could be delivered to the new line. Given the serious nature of these considerations, an alternative extraction scheme has been proposed based on laser neutralization.

Laser extraction addresses all three of the above concerns. Only the amount of beam required would need to be extracted to the new line, eliminating the problem of what to do with the full Linac intensity. Given that only a fraction, $1-2 \%$, of the beam would be diverted, and that the remaining beam could be injected into the Booster, this scheme is compatible with accelerator operations. (The 1-2\% figure was obtained by assuming $10 \%$ stripping of chopper-deflected beam for $2 \mu \mathrm{sec}$ out of 10 , which corresponds to 4-5 turn Booster injection at $400 \mathrm{MeV}$.) The top-end power of the laser would provide an inherent safety device automatically placing a ceiling on the total amount of beam that could possibly be delivered to the new line. 


\section{Acknowledgements}

I wish to thank Fred Mills and Arlene Lennox for their useful discussions on this subject. Arlene, in particular, contributed to the final version of this paper.

\section{References}

1. M. Popovic, private communication.

2. "Proton Therapy at the SSC," Conceptual Design Summary prepared for and work supported by Southwestern Medical Center, SSC internal report, April, 1992.

3. E. Hubbard, "Booster Synchrotron," Fermilab Technical Memo TM-405.

4. C. Schmidt, private communication.

5. P. H. Crandall, C. F. Burnett, "Fundamental Atomic Collisional Processes in Negative Ion Sources for $H^{-}$," BNL 50727 UC-344, 1977, pg. 185.

6. F. Mills, private communication. 


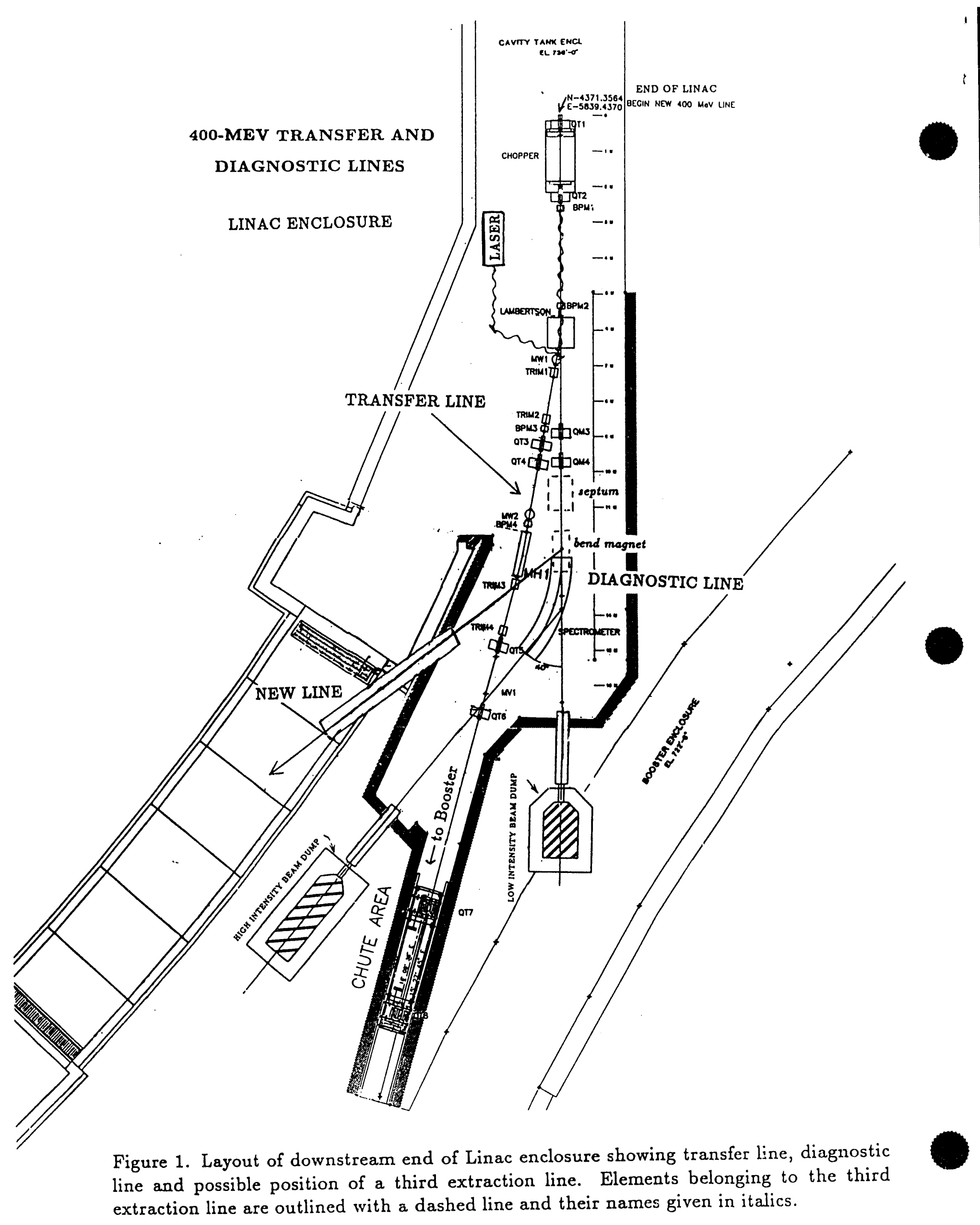



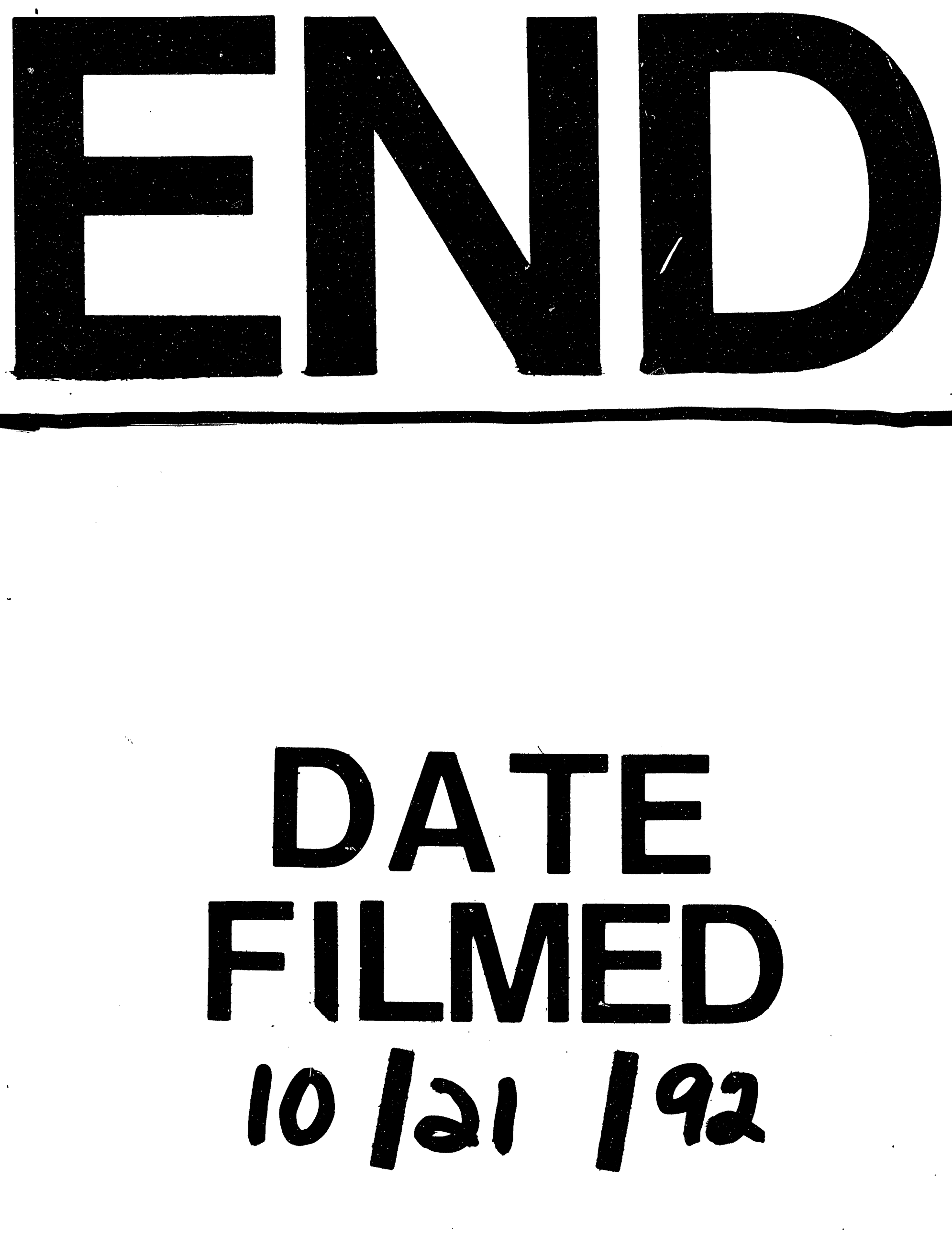


\section{เ}

